\title{
Analysis of Two Attacks on Reduced-Round Versions of the SMS4
}

\author{
Deniz Toz ${ }^{1}$ and Orr Dunkelman ${ }^{2,3}$ \\ ${ }^{1}$ Middle East Technical University \\ Institute of Applied Mathematics, Ankara, Turkey \\ deniz.toz@gmail.com \\ ${ }^{2}$ Katholieke Universiteit Leuven \\ Department of Electronical Engineering ESAT SDC-COSIC \\ and \\ Interdisciplinary Institute for BroadBand Technology (IBBT) \\ Kasteelpark Arenberg 10, B-3001 Leuven-Heverlee, Belgium \\ orr.dunkelman@esat.kuleuven . be \\ ${ }^{3}$ École Normale Supérieure \\ Département d'Informatique, \\ CNRS, INRIA \\ 45 rue d'Ulm, 75230 Paris, France
}

\begin{abstract}
SMS4 is a 128-bit block cipher used in WAPI (the Chinese national standard for wireless networks). Up until recently, the best attacks on SMS4 known, in terms of the number of rounds, were the rectangle attack on 14 rounds and the impossible differential attack on 16 rounds (out of 32 rounds) presented by Lu. While analyzing them, we noticed that these attacks have flaws and that their complexity analysis is inaccurate. In this paper we make a more comprehensive analysis of these attacks and further improve these results.
\end{abstract}

\section{Introduction}

SMS4 1 is a Generalized Feistel Network (GFN) cipher, specified in the Wireless Authentication and Privacy Infrastructure (WAPI), which is mandatory in wireless networks in China. The cipher has block size of 128 bits, and each block is processed in 32 rounds using a secret key of 128 bits long.

The Chinese Standards Association (SAC) submitted WAPI to ISO for recognition as an international standard, at about the same time as the IEEE 802.11i standard. As a result, SMS4 was the subject of an extensive international debate since its introduction. Despite that, up until recently little cryptanalysis of SMS4 was performed. The previously published cryptanalytic results are the differential fault analysis presented in [11, the integral attack on 13 rounds 8, the rectangle attack on 14 rounds and the impossible differential attack on 16 rounds 9], the rectangle attack on 16 rounds and the differential attack on 21 rounds 12 , and finally the rectangle attack on 18 rounds, the differential attack and linear attack on 22 rounds 6$]^{1}$.

${ }^{1}$ We note that the results in $6[12$ were found independently of our line of research.

L. Chen, M.D. Ryan, and G. Wang (Eds.): ICICS 2008, LNCS 5308, pp. 141-156 2008.

(C) Springer-Verlag Berlin Heidelberg 2008 
The cryptanalytic results on SMS4, on which we focus are those of [9]. The proposed rectangle attack on 14 rounds of SMS4 uses $2^{121.82}$ chosen plaintexts and has a claimed time complexity of $2^{116.66} 14$-round SMS4 computations2. The impossible differential attack on 16-round SMS4 from 9] uses $2^{105}$ chosen plaintexts and its time complexity is conjectured to be $2^{107} 16$-round SMS4 computations.

While verifying the results of [9], we found several flaws and possible improvements. In this paper, we show that the actual probability of the 12-round rectangle distinguishers of 9 is $2^{-230.71}$, rather than the claimed probability of $2^{-237.64}$. We also present better 12 -round rectangle distinguishers with probability of $2^{-209.78}$. Moreover, we show that the claimed time complexity of the rectangle attack of [9] is flawed due to the deficient process of obtaining candidate quartets, which is not considered in the original time complexity analysis. Therefore, given our improved distinguishers and refined analysis, we present a 14-round rectangle attack that uses $2^{106.89}$ chosen plaintexts pairs and has running time of $2^{107.89}$ encryptions for obtaining the data, $2^{107.89}$ memory accesses to find the pairs, and $2^{87.97}$ encryptions for the analysis. Similarly, we identify several flaws in the impossible differential attack of [9]. We first show that more data is needed than the claimed figures, and then we point out a delicate issue concerning the running time of this attack. We then follow to suggest a corrected attack with data complexity of $2^{117.06}$ chosen plaintexts and time complexity of $2^{117.06}$ encryptions for obtaining the data, $2^{132.06}$ memory accesses for the preliminary elimination, and $2^{95.09}$ encryptions for the analysis.

Independent of our research, SMS4 was analyzed also in 612. A rectangle attack on 16 rounds of SMS4 which requires $2^{124}$ chosen plaintexts with a time complexity of $2^{116}$ encryptions, and a differential attack on 21 rounds of SMS4 with data and time complexities of $2^{118}$ and $2^{112.83}$, respectively are presented in 12 . These results are improved in 6, by using the early abort technique, to a rectangle attack on 18 rounds of SMS4 with a data complexity of $2^{120}$ and time complexity of $2^{116.83}$, a differential attack on 22 rounds of SMS4 with a data complexity of $2^{118}$ chosen plaintexts, and a time complexity of $2^{125.71}$ encryptions. Also, a linear attack on 22 rounds of SMS4 which has data complexity of $2^{117}$ known plaintexts, and time complexity of $2^{109.86}$ encryptions is described in 6.

This paper is organized as follows: In Section 2, we give a brief description of the SMS4 cipher and its properties. In Section 3, we give an overview of the rectangle attack, followed by the previous rectangle attack of [9] on SMS4. Then, we present our observations and improvements for this attack on SMS4. In Section 4 we follow the same outline for the impossible differential attack. Finally, we conclude this paper and summarize our findings in Section 5 .

\footnotetext{
${ }^{2}$ This is the claimed time complexity in 9, but in fact the actual number should be $2^{121.82}$, which is the time required to obtain the ciphertexts to perform attack, according to [9].
} 


\section{A Description of SMS4}

\subsection{Notation}

Throughout this paper, we will use the following notation. Each 128-bit block is composed of four 32-bit words $\left(X_{0}, X_{1}, X_{2}, X_{3}\right)$. Note that the words and blocks are in a "Chinese"-endian order (i.e., the most significant bit is the leftmost bit numbered 0, and the least significant bit is bit 31 for a 32-bit word). Similarly, the most significant byte of a word is the leftmost byte numbered 0 , and least significant byte is numbered 3. We denote the bit rotation of the word $w$ by $r$ positions to the left by $w \lll r ; e_{j}$ denotes a word whose all positions except the $j$-th bit are zero and

$$
e_{i_{1}, \ldots, i_{j}}=e_{i_{1}} \oplus \ldots \oplus e_{i_{j}} \text { for } 0 \leq i_{1}, \ldots, i_{j} \leq 31
$$

\subsection{The SMS4 Cipher}

SMS4 [1] accepts a 128-bit plaintext $P=\left(P_{0}, P_{1}, P_{2}, P_{3}\right)$ and a 128-bit user key as inputs, and is composed of 32 rounds. In each round, the least significant three bytes of the state are xored with the round key and the result passes the $\mathrm{S}$ transformation. The $\mathrm{S}$ transformation uses an 8-bit to 8-bit bijective SBox four times in parallel to process each byte, then the concatenated bytes are processed using a linear transformation $L$. Let $X_{i}=\left(X_{i, 0}, X_{i, 1}, X_{i, 2}, X_{i, 3}\right)$ and $X_{i+1}=\left(X_{i+1,0}, X_{i+1,1}, X_{i+1,2}, X_{i+1,3}\right)$ denote the 128 -bit input and output to the $i$-th round, respectively. Then the round function may be formally described by the following equations:

$$
\begin{aligned}
& X_{i+1,0}=X_{i, 1} \\
& X_{i+1,1}=X_{i, 2} \\
& X_{i+1,2}=X_{i, 3} \\
& X_{i+1,3}=X_{i+1,0} \oplus L\left(S\left(X_{i, 1} \oplus X_{i, 2} \oplus X_{i, 3} \oplus R K_{i}\right)\right)
\end{aligned}
$$

where the $\mathrm{S}$ transformation uses the SBox given in [1] and $L$ is the linear transformation:

$$
L(x)=x \oplus(x \lll 2) \oplus(x \lll 10) \oplus(x \lll 18) \oplus(x \lll 24) \text { where } x \in Z_{2}^{32}
$$

The transformation $L \circ S$ is named $T$ in the specification document. $R K_{i}$ is the 32-bit round sub key for the $i$-th round, obtained from the key schedule. Decryption is identical to the encryption except for the order of the subkeys, which are used in the reverse order.

Key Schedule: The key schedule is similar to the encryption function. The only difference is that instead of using the linear transformation $L$, the following linear transformation $L^{\prime}$ is used:

$$
L^{\prime}(x)=x \oplus(x \lll 13) \oplus(x \lll 23) \text { where } x \in Z_{2}^{32}
$$




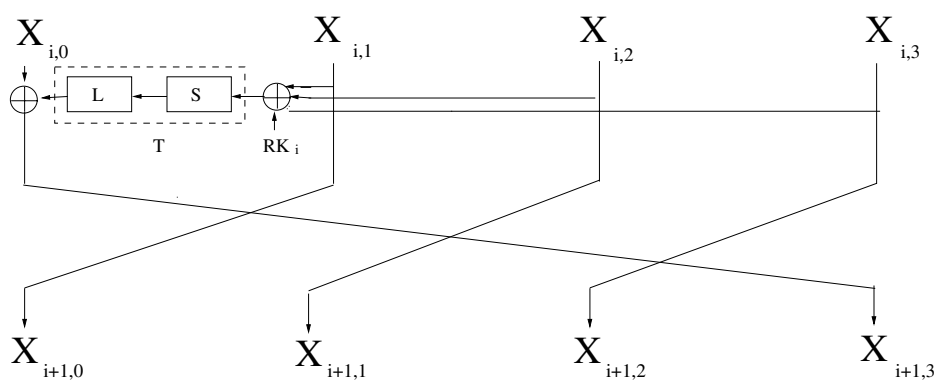

Fig. 1. Round Function

In addition, the user supplied key $K$ is xored with a system parameter, $F K$. The subkey $R K_{j}$ of the $j$-th round is computed as follows:

$$
\begin{aligned}
F K & =(0 x A 3 B 1 B A C 6,0 x 56 A A 3350,0 x 677 D 9197,0 x B 27022 D C) \\
k & =\left(k_{0}, k_{1}, k_{2}, k_{3}\right)=K \oplus F K \\
R K_{j} & =k_{j+4}=k_{j} \oplus L^{\prime}\left(S\left(k_{j+1} \oplus k_{j+2} \oplus k_{j+3} \oplus C K_{j}\right)\right)
\end{aligned}
$$

where $C K_{j}=\left(c k_{j, 0}, c k_{j, 1}, c k_{j, 2}, c k_{j, 3}\right)$ and $c k_{j, k}=28 j+7 k(\bmod 256)$.

\subsection{Properties and Definitions}

Since SMS4 uses a bijective SBox, thus, $S(\Delta x)=0$ if and only if $\Delta x=0$. The difference distribution table (DDT) of the SBox contains exactly 127 nonzero output differences for a given nonzero input difference. Only one of these values has probability of $2^{-6}$ while the other 126 remaining nonzero values have probability of $2^{-7}$.

The following definitions are used for observing the propagation of any nonzero input difference to the other rounds. In [9], it is not clearly stated to what these sets refer, and the formulas contain typos. Thus, the reader may find the original terminology confusing. Therefore, we rewrite the equations defining these sets, using the same names for the sets (but with a clearer representation).

Given the input difference $\left(0, e_{\Lambda}, e_{\Lambda}, e_{\Lambda}\right)$ to the $n$-th round, where $\Lambda$ is an arbitrary but nonempty subset of $\{0,1, \ldots, 31\}$, the set $\theta\left(e_{\Lambda}\right)$ is composed of all the 32-bit differences that an input difference $e_{\Lambda}$ to the $\mathrm{T}$ function can cause:

$$
\theta\left(e_{\Lambda}\right)=\left\{x \mid x=L\left(\Delta d_{1}\right), \operatorname{Pr}\left[S\left(e_{\Lambda}\right) \rightarrow \Delta d_{1}\right]>0 \text { for } x, e_{\Lambda} \in Z_{2}^{32}\right\}
$$

Now, the input difference to the $(n+1)$-th round is $\left(e_{\Lambda}, e_{\Lambda}, e_{\Lambda}, X\right)$ where $X \in$ $\theta\left(e_{\Lambda}\right)$. The $\Upsilon\left(e_{\Lambda}, X\right)$ is the set of all 32-bit differences, that an input difference $X$ to the $\mathrm{T}$ function may cause after an xor with $e_{\Lambda}$.

$$
\Upsilon\left(e_{\Lambda}, X\right)=\left\{y \mid y=L\left(\Delta d_{2}\right) \oplus e_{\Lambda}, \operatorname{Pr}\left[S(X) \rightarrow \Delta d_{2}\right]>0 \text { for } X \in \theta\left(e_{\Lambda}\right), y, e_{\Lambda} \in Z_{2}^{32}\right\}
$$

Similarly, the input difference to the $(n+2)$-th round is of the form $\left(e_{\Lambda}, e_{\Lambda}, X, Y\right)$ where $X \in \theta\left(e_{\Lambda}\right)$ and $Y \in \Upsilon\left(e_{\Lambda}, X\right)$. The corresponding 32-bit output differences, caused by an input difference $e_{\Lambda} \oplus X \oplus Y$ to $\mathrm{T}$ are denoted by the set 


$$
\begin{aligned}
& \Pi\left(e_{\Lambda}, X, Y\right) . \\
& \Pi\left(e_{\Lambda}, X, Y\right)=\left\{z \mid z=L\left(\Delta d_{3}\right) \oplus e_{\Lambda}, \operatorname{Pr}\left[S\left(e_{\Lambda} \oplus X \oplus Y\right) \rightarrow \Delta d_{3}\right]>0 \text { for } z, e_{\Lambda} \in Z_{2}^{32}\right\}
\end{aligned}
$$

Finally, the input difference to the $(n+3)$-th round is of the form $\left(e_{\Lambda}, X, Y, Z\right)$ where $X \in \theta\left(e_{\Lambda}\right), Y \in \Upsilon\left(e_{\Lambda}, X\right)$, and $Z \in \Pi\left(e_{\Lambda}, X, Y\right)$. The set of 32-bit differences after the XOR operation in the $(n+3)$-th round by an input difference $X \oplus Y \oplus Z$ to $\mathrm{T}$ is denoted by the set $\Omega(X, Y, Z)$.

$\Omega(X, Y, Z)=\left\{w \mid w=L\left(\Delta d_{4}\right) \oplus e_{\Lambda}, \operatorname{Pr}\left[S(X \oplus Y \oplus Z) \rightarrow \Delta d_{4}\right]>0\right.$ for $\left.w \in Z_{2}^{32}\right\}$

\section{The Rectangle Attack}

The amplified boomerang and rectangle attacks [2] are a chosen plaintext attacks, which evolved from the boomerang attack 10. The main idea in these attacks is to use two short differential characteristics with high probabilities instead of one long characteristic with a lower probability. The only difference is that the boomerang attack generates a quartet at an intermediate value halfway through the cipher, whereas the rectangle attack looks for quartets within a given set of pairs.

For this purpose, the block cipher $E$ is treated as a cascade of two sub-ciphers $E_{0}$ and $E_{1}$ (i.e, $E=E_{1} \circ E_{0}$ ). Assume that a differential characteristics $\Delta \rightarrow \Delta^{*}$ with probability $p$ for $E_{0}$, and $\nabla^{*} \rightarrow \nabla$ with probability $q$ for $E_{1}$ are known. The boomerang attack is based on generating right quartets $\left(P_{1}, P_{2}, P_{3}, P_{4}\right)$ which satisfy a set of relations:

1. $P_{1} \oplus P_{2}=\Delta=P_{3} \oplus P_{4}$.

2. $E_{0}\left(P_{1}\right) \oplus E_{0}\left(P_{2}\right)=\Delta^{*}=E_{0}\left(P_{3}\right) \oplus E_{0}\left(P_{4}\right)$.

3. $E_{0}\left(P_{1}\right) \oplus E_{0}\left(P_{3}\right)=\nabla^{*}=E_{0}\left(P_{2}\right) \oplus E_{0}\left(P_{4}\right)$.

4. $C_{1} \oplus C_{3}=\nabla=C_{2} \oplus C_{4}$ where $C_{i}=E_{1}\left(E_{0}\left(P_{i}\right)\right)$.

A right quartet which satisfies the above equations is formed as follows:

1. Choose a random plaintext $P_{1}$ and compute $P_{2}=P_{1} \oplus \Delta$.

2. Ask for the encryptions of $P_{1}$ and $P_{2}$ to obtain $C_{1}=E\left(P_{1}\right)$ and $C_{2}=E\left(P_{2}\right)$.

3. Calculate $C_{3}=C_{1} \oplus \nabla$ and $C_{4}=C_{2} \oplus \nabla$.

4. Ask for the decryptions of $C_{3}$ and $C_{4}$ to obtain $P_{3}=D\left(C_{3}\right)$ and $P_{4}=D\left(C_{4}\right)$.

5. Check whether $P_{3} \oplus P_{4}=\Delta$.

The amplified boomerang attack is a chosen plaintext attack in which the same differential conditions have to be satisfied. But instead of generating quartets as given above, a set of plaintext pairs with input difference $\Delta$ is generated. Then the aim is to find quartets $\left(\left(P_{1}, P_{2}\right),\left(P_{3}, P 4\right)\right)$ such that $C_{1} \oplus C_{3}=\nabla=C_{2} \oplus C_{4}$ when $P_{1} \oplus P_{2}=\Delta=P_{3} \oplus P_{4}$ by using birthday paradox.

By a more careful analysis and a better key recovery algorithm, the amplified boomerang attack was evolved into the rectangle attack. For an optimized method of finding the right rectangle quartet, one may refer to [3]. 
In [210], it is shown that it is possible to use all possible $\Delta^{*}$ 's and $\nabla^{*}$ 's simultaneously. In [2, it is also stated that, if $N$ plaintext pairs with input difference $\Delta$, then the number of expected right quartets is $N^{2} 2^{-128} \hat{p}^{2} \hat{q}^{2}$ for 128-bit block ciphers, where

$$
\hat{p}=\sqrt{\sum_{\Delta^{*}} \operatorname{Pr}^{2}\left[\Delta \rightarrow \Delta^{*}\right]} \quad \text { and } \hat{q}=\sqrt{\sum_{\nabla} \operatorname{Pr}^{2}\left[\nabla^{*} \rightarrow \nabla\right]}
$$

\subsection{The Rectangle Attack on 14-Round SMS4 from [9]}

The 14-round rectangle attack in [9] uses 12-round rectangle distinguishers with probability $2^{-230.71}$ and requires $2^{121.82}$ chosen plaintexts to attack 14 -round SMS4. Let $E_{0}$ denote rounds 0 to 7 and let $E_{1}$ denote rounds 8 to 11 of SMS4. The differentials used for the 12-round distinguishers of [9] are as follows:

1. For $E_{0}$ : All 8-round differentials of the form $\left(e_{\psi_{1}}, e_{\psi}, e_{\psi}, e_{\psi}\right) \rightarrow\left(e_{\psi_{2}}, e_{\psi_{3}}, e_{\psi_{4}}, e_{\psi_{5}}\right)$ where only one byte of $e_{\psi}$ is nonzero and $e_{\psi_{1}}, e_{\psi_{2}} \in \theta\left(e_{\psi}\right), e_{\psi_{3}} \in \Upsilon\left(e_{\psi}, e_{\psi_{2}}\right)$, $e_{\psi_{4}} \in \Pi\left(e_{\psi}, e_{\psi_{2}}, e_{\psi_{3}}\right), e_{\psi_{5}} \in \Omega\left(e_{\psi_{2}}, e_{\psi_{3}}, e_{\psi_{4}}\right)$, and $e_{\psi_{1}}$ is fixed.

2. For $E_{1}$ : All 4-round differentials of the form $\left(e_{\Phi}, e_{\Phi}, e_{\Phi}, 0\right) \rightarrow\left(e_{\Phi}, e_{\Phi}, e_{\Phi}, e_{\Phi_{2}}\right)$ where only one byte of $e_{\Phi}$ is nonzero and $e_{\Phi_{2}} \in \theta\left(e_{\Phi}\right)$.

To calculate the overall probability, the sum of the squares of the probabilities of all used differentials is needed. As there are many 8-round differential characteristics, we list the ones that follow the path in Table 1. In Table 2 we list how many differential characteristics of a given probability follow this path.

Therefore, the lower bound can be calculated as:

$$
\begin{aligned}
\hat{p}^{2} & =\left(2^{-6}\right)^{2} \cdot\left[\left(2^{-6}\right)^{2}+126 \cdot\left(2^{-7}\right)^{2}\right] \cdot\left[\left(2^{-24}\right)^{2}+\left(\begin{array}{l}
4 \\
3
\end{array}\right) \cdot 126 \cdot\left(2^{-25}\right)^{2}\right. \\
& \left.+\left(\begin{array}{l}
4 \\
2
\end{array}\right) \cdot 126^{2} \cdot\left(2^{-26}\right)^{2}+\left(\begin{array}{l}
4 \\
1
\end{array}\right) \cdot 126^{3} \cdot\left(2^{-27}\right)^{2}+126^{4} \cdot\left(2^{-28}\right)^{2}\right]^{3} \\
& =2^{-102.71}
\end{aligned}
$$

We note that the second differential is a truncated differential with 127 possible output differences and probability one. Therefore:

$$
\hat{q}^{2}=1
$$

Thus, the expected number of right rectangle quartets generated by $\mathrm{N}$ plaintext pairs is:

$$
N^{2} \cdot 2^{-128} \cdot \hat{p}^{2} \cdot \hat{q}^{2}=N^{2} \cdot 2^{-230.71}
$$

Attack Procedure: The above 12-round distinguishers are used to mount a rectangle attack on 14-round SMS4. Given the 127 input differences $\left(e_{\Phi}, e_{\Phi}\right.$,

\footnotetext{
${ }^{3}$ In 9 the probability of these 12-round distinguishers is calculated as $2^{-237.64}$ due to a miscalculation of $\hat{q}$.

${ }^{4}$ This is the output difference of the distinguisher, and $e_{\Phi_{2}}$ can take any value (of the 127 possible ones). Thus, the probability of the last step is one. In 9], probability is also calculated for this step, leading to a faulty, lower, probability for the distinguisher.
} 
Table 1. The number of differences and their probabilities for the 8-round characteristic

\begin{tabular}{|c|c|c|c|c|c|c|c|c|}
\hline$e_{\psi_{1}}$ & (for & $\begin{array}{l}\psi_{2} \\
\left.\text { ixed } e_{\psi}\right)\end{array}$ & $\begin{array}{r}e_{\psi} \\
\text { (for a giv } \\
\end{array}$ & $\left.n e_{\psi_{2}}\right)$ & $\begin{array}{r}e \\
\text { (for a give }\end{array}$ & $\left.e_{\psi_{2}}, e_{\psi_{3}}\right)$ & (for a giv & $\begin{array}{l}e_{\psi_{5}} \\
\left.e_{\psi_{2}}, e_{\psi_{3}}, e_{\psi_{4}}\right)\end{array}$ \\
\hline No $\mathrm{Pr}$ & No & $\mathrm{Pr}$ & No & $\mathrm{Pr}$ & $\mathrm{No}$ & $\mathrm{Pr}$ & No & $\mathrm{Pr}$ \\
\hline $12^{-6}$ & $\begin{array}{c}1 \\
126\end{array}$ & $\begin{array}{l}2^{-6} \\
2^{-7}\end{array}$ & $\begin{array}{c}1 \\
\left(\begin{array}{l}4 \\
3\end{array}\right) \cdot 126 \\
\left(\begin{array}{l}4 \\
2\end{array}\right) \cdot 126^{2} \\
\left(\begin{array}{l}4 \\
1\end{array}\right) \cdot 126^{3} \\
126^{4}\end{array}$ & $\begin{array}{l}2^{-24} \\
2^{-25} \\
2^{-25} \\
2^{-27} \\
2^{-28}\end{array}$ & $\begin{array}{c}1 \\
\left(\begin{array}{c}4 \\
3\end{array}\right) \cdot 126 \\
\left(\begin{array}{l}4 \\
2\end{array}\right) \cdot 126^{2} \\
\left(\begin{array}{l}4 \\
1 \\
1\end{array}\right) \cdot 126^{3} \\
126^{4}\end{array}$ & $\begin{array}{l}2^{-24} \\
2^{-25} \\
2^{-25} \\
2^{-27} \\
2^{-28}\end{array}$ & $\begin{array}{c}1 \\
\left(\begin{array}{l}4 \\
3\end{array}\right) \cdot 126 \\
\left(\begin{array}{l}4 \\
2\end{array}\right) \cdot 126^{2} \\
\left(\begin{array}{l}4 \\
1\end{array}\right) \cdot 126^{3} \\
126^{4}\end{array}$ & $\begin{array}{l}2^{-24} \\
2^{-25} \\
2^{-25} \\
2^{-27} \\
2^{-28}\end{array}$ \\
\hline
\end{tabular}

Table 2. The number of characteristics and their probabilities for $E_{0}$

\begin{tabular}{|l|c|c|c|c|c|c|c|}
\hline Probability & $2^{-84}$ & $2^{-85}$ & $2^{-86}$ & $2^{-87}$ & $2^{-88}$ & $2^{-89}$ & $2^{-90}$ \\
Number & 1 & $2^{10.678}$ & $2^{20.312}$ & $2^{29.217}$ & $2^{37.514}$ & $2^{45.277}$ & $2^{52.561}$ \\
\hline Probability & $2^{-91}$ & $2^{-92}$ & $2^{-93}$ & $2^{-94}$ & $2^{-95}$ & $2^{-96}$ & $2^{-97}$ \\
Number & $2^{59.411}$ & $2^{65.872}$ & $2^{71.972}$ & $2^{77.692}$ & $2^{82.920}$ & $2^{87.428}$ & $2^{90.704}$ \\
\hline
\end{tabular}

$\left.e_{\Phi}, e_{\Phi_{2}}\right)$ to round 12 , there are $127^{5}$ possible output differences $\left(e_{\Phi}, e_{\Phi}, e_{\Phi_{2}}, e_{\Phi_{3}}\right)$ just after round 12 , where $e_{\Phi_{3}} \in \Upsilon\left(e_{\Phi}, e_{\Phi_{2}}\right)$ and $127^{9}$ possible output differences $\left(e_{\Phi}, e_{\Phi_{2}}, e_{\Phi_{3}}, e_{\Phi_{4}}\right)$, where $e_{\Phi_{4}} \in \Pi\left(e_{\Phi}, e_{\Phi_{2}}, e_{\Phi_{3}}\right)$. For sake of clarity, we define all these output differences by the set $\Phi$ :

$$
\Phi=\left\{\left(e_{\Phi}, e_{\Phi_{2}}, e_{\Phi_{3}}, e_{\Phi_{4}}\right) \mid e_{\Phi_{2}} \in \Theta\left(e_{\Phi}\right), e_{\Phi_{3}} \in \Upsilon\left(e_{\Phi}, e_{\Phi_{2}}\right), e_{\Phi_{4}} \in \Pi\left(e_{\Phi}, e_{\Phi_{2}}, e_{\Phi_{3}}\right)\right\}
$$

The proposed attack uses an early abort technique, which allows partially determining whether or not a candidate quartet is a right one by guessing only a small fraction of the subkey, and if not discarding the quartet.

The attack procedure of [9] is as follows:

1. Choose $2^{120.82}$ pairs of plaintexts $5\left(P_{i}, P_{i}^{\prime}\right)$ with input difference $\left(e_{\psi_{1}}, e_{\psi}\right.$, $\left.e_{\psi}, e_{\psi}\right)$

(a) Obtain the corresponding ciphertext pairs $\left(C_{i}, C_{i}^{\prime}\right)$.

(b) Generate all candidate quartets $\left(\left(C_{i_{1}}, C_{i_{1}}^{\prime}\right),\left(C_{i_{2}}, C_{i_{2}}^{\prime}\right)\right)$.

(c) Check whether $C_{i_{1}} \oplus C_{i_{2}} \in \Phi$ and $C_{i_{1}}^{\prime} \oplus C_{i_{2}}^{\prime} \in \Phi$.

2. For each remaining quartet $\left(\left(C_{i_{1}}, C_{i_{1}}^{\prime}\right),\left(C_{i_{2}}, C_{i_{2}}^{\prime}\right)\right)$ :

(a) For each pair $\left(\left(C_{i_{1}}, C_{i_{1}}^{\prime}\right)\right.$ and $\left.\left(C_{i_{2}}, C_{i_{2}}^{\prime}\right)\right)$ compute the differences in the 4 bytes of their intermediate values just before the $L$ transformation in round 13 , and denote them by $\Delta_{i_{1}, i_{2}}^{13}$ and $\Delta_{i_{1}, i_{2}}^{\prime 13}$, respectively. (i.e, compute $\Delta_{i_{1}, i_{2}}^{13}=L^{-1}\left(C_{i_{1}} \oplus C_{i_{2}}\right)$ and $\Delta_{i_{1}, i_{2}}^{\prime}=L^{-1}\left(C_{i_{1}}^{\prime} \oplus C_{i_{2}}^{\prime}\right)$.

(b) For $j=0$ to 3 :

${ }^{5}$ The required number of plaintext pairs is not adapted for the new probability, since it has no effect on our findings. 
i. Guess the $j$-th byte of the subkey $R K_{13}$ and partially decrypt every remaining quartet to obtain the $j$-th byte of their intermediate values just after the $\mathrm{S}$ transformation in round 13. Denote them by $\left(\left(X_{i_{1}, j}, X_{i_{2}, j}\right),\left(X_{i_{1}, j}^{\prime}, X_{i_{2}, j}^{\prime}\right)\right)$.

ii. Check if $X_{i_{1}, j} \oplus X_{i_{2}, j}=\Delta_{i_{1}, i_{2}, j}^{13}$ and $X_{i_{1}, j}^{\prime} \oplus X_{i_{2}, j}^{\prime}=\Delta_{i_{1} . i_{2}, j}^{\prime 13}$ and keep only the quartets for which both equalities are satisfied.

3. For each remaining quartet $\left(\left(T_{i_{1}}, T_{i_{1}}^{\prime}\right),\left(T_{i_{2}}, T_{i_{2}}^{\prime}\right)\right)$ repeat Step 2 , for round 12 and $R K_{12}$.

4. If for a subkey guess $\left(R K_{12}, R K_{13}\right)$, there are 6 (or more) remaining quartets try all possible $\left(R K_{10}, R K_{11}\right)$ values and perform a trial encryption with one known plaintext/ciphertext pair. If the correct key is not found for all checked $\left(R K_{12}, R K_{13}\right)$ values output "failure".

\subsection{Improving the 14-Round Attack}

By a simple observation, one can conclude that $E_{0}$ in the 14-round attack has too many rounds. After round 3, each additional round comes with a cost (in terms of probability) increasing exponentially. Therefore, the attack can be improved by using a shorter characteristic for $E_{0}$ with higher probability in exchange for making $E_{1}$ longer. We suggest the use of following differential characteristics:

1. For $E_{0}$ : The 6-round differentials $\left(e_{\psi_{1}}, e_{\psi}, e_{\psi}, e_{\psi}\right) \rightarrow\left(e_{\psi}, e_{\psi}, e_{\psi_{2}}, e_{\psi_{3}}\right)$ where only one byte of $e_{\psi}$ is nonzero, $e_{\psi_{1}}, e_{\psi_{2}} \in \theta\left(e_{\psi}\right), e_{\psi_{3}} \in \Upsilon\left(e_{\psi}, e_{\psi_{2}}\right)$, and $e_{\psi_{1}}$ is fixed.

2. For $E_{1}$ : The 6-round differentials $\left(e_{\Phi_{6}}, e_{\Phi_{5}}, e_{\Phi}, e_{\Phi}\right) \rightarrow\left(e_{\Phi}, e_{\Phi}, e_{\Phi}, e_{\Phi_{2}}\right)$ where only one byte of $e_{\Phi}$ is nonzero and $e_{\Phi_{5}}, e_{\Phi_{2}} \in \theta\left(e_{\Phi}\right), e_{\Phi_{6}} \in \Upsilon\left(e_{\Phi}, e_{\Phi_{5}}\right)$.

The details of the rectangle distinguishers for the original attack and the proposed improvement are given in Table 3 .

The probability of the new proposed distinguisher can be calculated as follows:

As mentioned earlier in Section 3.1, there exists one possible $e_{\psi_{2}}$ with probability $2^{-6}$ and 126 possible $e_{\psi_{2}}$ values with probability $2^{-7}$ in round 4 . And in round 5 , for each of the $e_{\psi_{2}}$ values, we have one possible $e_{\psi_{3}}$ with probability $2^{-24},\left(\begin{array}{l}4 \\ 1\end{array}\right) \times 126$ possible values with probability $2^{-25},\left(\begin{array}{l}4 \\ 2\end{array}\right) \times 126^{2}$ possible values with probability $2^{-26},\left(\begin{array}{l}4 \\ 1\end{array}\right) \times 126^{3}$ possible values with probability $2^{-27}$ and $126^{4}$ possible values with probability $2^{-28}$. Hence for $E_{0}$, we have:

$$
\begin{aligned}
\hat{p}^{2} & =\left(2^{-6}\right)^{2} \cdot\left[\left(2^{-6}\right)^{2}+126 \cdot\left(2^{-7}\right)^{2}\right] \cdot\left[\left(2^{-24}\right)^{2}+\left(\begin{array}{l}
4 \\
3
\end{array}\right) \cdot 126 \cdot\left(2^{-25}\right)^{2}\right. \\
& \left.+\left(\begin{array}{l}
4 \\
2
\end{array}\right) \cdot 126^{2} \cdot\left(2^{-26}\right)^{2}+\left(\begin{array}{l}
4 \\
1
\end{array}\right) \cdot 126^{3} \cdot\left(2^{-27}\right)^{2}+126^{4} \cdot\left(2^{-28}\right)^{2}\right] \\
& =2^{-46.8881}
\end{aligned}
$$

Similarly, for $E_{1}$, in round 7 , we have one possible $e_{\Phi_{5}}$ with probability $2^{-6}$ and 126 possible $e_{\Phi_{5}}$ with probability $2^{-7}$. In round 6 , for each of the $e_{\Phi_{5}}$ values, there is one possible $e_{\Phi_{6}}$ with probability $2^{-24},\left(\begin{array}{l}4 \\ 1\end{array}\right) \times 126$ possible values with probability $2^{-25},\left(\begin{array}{l}4 \\ 2\end{array}\right) \times 126^{2}$ possible values with probability $2^{-26},\left(\begin{array}{l}4 \\ 1\end{array}\right) \times 126^{3}$ 
Table 3. The rectangle attack distinguishers

\begin{tabular}{|c|c|c|c|c|c|c|c|c|c|c|c|}
\hline \multicolumn{6}{|c|}{ Previous Attack } & \multicolumn{6}{|c|}{ Improved Attack } \\
\hline Round & $\Delta X_{i, 0}$ & $\Delta X_{i, 1}$ & $\Delta X_{i, 2}$ & $\Delta X_{i .3}$ & Prob & Round & $\Delta X_{i, 0}$ & $\Delta X_{i, 1}$ & $\Delta X_{i, 2}$ & $\Delta X_{i, 3}$ & Prob \\
\hline 0 & $e_{\psi_{1}}$ & $e_{\psi}$ & $e_{\psi}$ & $e_{\psi}$ & $2^{-6}$ & 0 & $e_{\psi_{1}}$ & $e_{\psi}$ & $e_{\psi}$ & $e_{\psi}$ & $2^{-6}$ \\
\hline 1 & $e_{\psi}$ & $e_{\psi}$ & $e_{\psi}$ & 0 & 1 & 1 & $e_{\psi}$ & $e_{\psi}$ & $e_{\psi}$ & 0 & 1 \\
\hline 2 & $e_{\psi}$ & $e_{\psi}$ & 0 & $e_{\psi}$ & 1 & 2 & $e_{\psi}$ & $e_{\psi}$ & 0 & $e_{\psi}$ & 1 \\
\hline 3 & $e_{\psi}$ & 0 & $e_{\psi}$ & $e_{\psi}$ & 1 & 3 & $e_{\psi}$ & 0 & $e_{\psi}$ & $e_{\psi}$ & 1 \\
\hline 4 & 0 & $e_{\psi}$ & $e_{\psi}$ & $e_{\psi}$ & $t^{a}$ & 4 & 0 & $e_{\psi}$ & $e_{\psi}$ & $e_{\psi}$ & $\dagger$ \\
\hline 5 & $e_{\psi}$ & $e_{\psi}$ & $e_{\psi}$ & $e_{\psi_{2}}$ & $\dagger$ & 5 & $e_{\psi}$ & $e_{\psi}$ & $e_{\psi}$ & $e_{\psi_{2}}$ & $\dagger$ \\
\hline 6 & $e_{\psi}$ & $e_{\psi}$ & $e_{\psi_{2}}$ & $e_{\psi_{3}}$ & $\dagger$ & output & $e_{\psi}$ & $e_{\psi}$ & $e_{\psi_{2}}$ & $e_{\psi_{3}}$ & \\
\hline 7 & $e_{\psi}$ & $e_{\psi_{2}}$ & $e_{\psi_{3}}$ & $e_{\psi_{4}}$ & $\dagger$ & 6 & $e_{\Phi_{6}}$ & $e_{\Phi_{5}}$ & $e_{\Phi}$ & $e_{\Phi}$ & $f^{b}$ \\
\hline output & $e_{\psi_{2}}$ & $e_{\psi_{3}}$ & $e_{\psi_{4}}$ & $e_{\psi_{5}}$ & & 7 & $e_{\Phi_{5}}$ & $e_{\Phi}$ & $e_{\Phi}$ & $e_{\Phi}$ & $\ddagger$ \\
\hline 8 & $e_{\Phi}$ & $e_{\Phi}$ & $e_{\Phi}$ & 0 & 1 & 8 & $e_{\Phi}$ & $e_{\Phi}$ & $e_{\Phi}$ & 0 & 1 \\
\hline 9 & $e_{\Phi}$ & $e_{\Phi}$ & 0 & $e_{\Phi}$ & 1 & 9 & $e_{\Phi}$ & $e_{\Phi}$ & 0 & $e_{\Phi}$ & 1 \\
\hline 10 & $e_{\Phi}$ & 0 & $e_{\Phi}$ & $e_{\Phi}$ & 1 & 10 & $e_{\Phi}$ & 0 & $e_{\Phi}$ & $e_{\Phi}$ & 1 \\
\hline 11 & 0 & $e_{\Phi}$ & $e_{\Phi}$ & $e_{\Phi}$ & 1 & 11 & 0 & $e_{\Phi}$ & $e_{\Phi}$ & $e_{\Phi}$ & 1 \\
\hline output & $e_{\Phi}$ & $e_{\Phi}$ & $e_{\Phi}$ & $e_{\Phi_{2}}$ & & output & $e_{\Phi}$ & $e_{\Phi}$ & $e_{\Phi}$ & $e_{\Phi_{2}}$ & \\
\hline
\end{tabular}

${ }^{a}$ The probabilities given with dagger are stated in Table 1.

${ }^{b}$ The probability of $e_{\Phi_{5}}$ is equal to the probability of $e_{\psi_{2}}$, since they both belong to the same set. Similarly $e_{\Phi_{6}}$ and $e_{\psi_{3}}$ have the same probability.

possible values with probability $2^{-27}$ and $126^{4}$ possible values with probability $2^{-28}$. Therefore:

$$
\begin{aligned}
\hat{q}^{2} & =\left[\left(2^{-6}\right)^{2}+126 \cdot\left(2^{-7}\right)^{2}\right] \cdot\left[\left(2^{-24}\right)^{2}+\left(\begin{array}{l}
4 \\
3
\end{array}\right) \cdot 126 \cdot\left(2^{-25}\right)^{2}+\left(\begin{array}{l}
4 \\
2
\end{array}\right) \cdot 126^{2} \cdot\left(2^{-26}\right)^{2}\right. \\
& \left.+\left(\begin{array}{l}
4 \\
1
\end{array}\right) \cdot 126^{3} \cdot\left(2^{-27}\right)^{2}+126^{4} \cdot\left(2^{-28}\right)^{2}\right] \\
& =2^{-34.8881}
\end{aligned}
$$

Thus, the expected number of right quartets generated by $\mathrm{N}$ plaintext pairs is:

$$
N^{2} \cdot 2^{-128} \cdot \hat{p}^{2} \cdot \hat{q}^{2}=N^{2} \cdot 2^{-209.78}
$$

In order to have sufficient pairs to perform the improved attack $N=2^{106.89}$ and from this point on we use this figure throughout the analysis.

A flaw in the preliminary elimination: In the original attack of [9], the time complexity is calculated only for candidates of right quartets after the preliminary elimination (the pairs which enter Step 2), and it does not include the time-complexity of the first elimination itself. However, due to the large amount of data, it is impossible to take all the possible pairs and detect candidates for right quartets immediately. We propose the following algorithm for the detection of right quartet candidates: 


\section{A more efficient algorithm for the preliminary elimination:}

1. Let $C_{i}=\left(C_{i, 0}, C_{i, 1}, C_{i, 2}, C_{i, 3}\right)$ and $C_{i}^{\prime}=\left(C_{i, 0}^{\prime}, C_{i, 1}^{\prime}, C_{i, 2}^{\prime}, C_{i, 3}^{\prime}\right)$ denote a ciphertext pair.

2. For each ciphertext pair $\left(C_{i}, C_{i}^{\prime}\right)$, insert the following entries into a hash table:

(a) $C_{i} \| C_{i}^{\prime}$ to the bin indexed by $C_{i, 0} C_{i, 1} \| C_{i, 0}^{\prime} C_{i, 1}^{\prime}$.

(b) $C_{i}^{\prime} \| C_{i}$ to the bin indexed by $C_{i, 0}^{\prime} C_{i, 1}^{\prime} \| C_{i, 0} C_{i, 1}$.

3. For every $\left(e_{\Phi_{2}}, e_{\Phi_{2}}^{\prime}\right)$ pair, where $e_{\Phi_{2}}, e_{\Phi_{2}}^{\prime} \in \Theta\left(e_{\Phi}\right)$ :

(a) initialize for each bin a flag to the state of "active".

(b) For every "active" bin satisfying $C_{i, 0} C_{i, 1} \leq C_{i, 0}^{\prime} C_{i, 1}^{\prime}$, go to the corresponding bin $C_{i, 0} C_{i, 1}\left\|C_{i, 0}^{\prime} C_{i, 1}^{\prime} \oplus e_{\Phi} e_{\Phi_{2}}\right\| e_{\Phi} e_{\Phi_{2}}^{\prime}=C_{j, 0} C_{j, 1} \| C_{j, 0}^{\prime} C_{j, 1}^{\prime}$.

i. For all possible combinations of entries $\left(\left(C_{i}, C_{i}^{\prime}\right),\left(C_{j}, C_{j}^{\prime}\right)\right)$, check whether:
A. $C_{i, 2} \oplus C_{j, 2} \in \Upsilon\left(e_{\Phi}, e_{\Phi_{2}}\right)$ and $C_{i, 2}^{\prime} \oplus C_{j, 2}^{\prime} \in \Upsilon\left(e_{\Phi}, e_{\Phi_{2}}^{\prime}\right)$
B. $C_{i, 3} \oplus C_{j, 3} \in \Pi\left(e_{\Phi}, e_{\Phi_{2}}, e_{\Phi_{3}}\right)$ and $C_{i, 2}^{\prime} \oplus C_{j, 2}^{\prime} \in \Pi\left(e_{\Phi}, e_{\Phi_{2}}^{\prime}, e_{\Phi_{3}}^{\prime}\right)$

(Once a condition fails, do not check the remaining conditions.)

ii. Flag the bins $C_{j, 0} C_{j, 1} \| C_{j, 0}^{\prime} C_{j, 1}^{\prime}$ and $C_{j, 0}^{\prime} C_{j, 1}^{\prime} \| C_{j, 0} C_{j, 1}$ as "analyzed".

4. If two pairs satisfying (i)-(ii) are found, keep them as candidates for right quartets, and apply steps $2-4$ of the attack in Section 3.1 .

If we have $\mathrm{N}$ pairs of ciphertexts, the expected number of entries in each of the $2^{128}$ bins is $2 \cdot N / 2^{128}=N \cdot 2^{-127}$ after Step 2. Therefore, we can form $127^{2} \cdot\left(N / 2^{127}\right)^{2}=N^{2} / 2^{240.02}$ candidate quartets for each pair of bins. Since we are only forming quartets for the bins whose first two words is smaller than its last two words, we analyze $2^{127}$ pairs of bins. Flagging in Step (b) also prevents analyzing the same quartet twice. So the number of candidate quartets entering Step (i) is $2^{127} / 2 \cdot N^{2} / 2^{240.02}=N^{2} / 2^{114.02}$. Now, the probability of passing Step $(\mathrm{A})$ is $\left(127^{4} / 2^{32}\right)^{2} \approx 2^{-8.08}$ and $2^{-8.08} \cdot N^{2} / 2^{114.02}=N^{2} / 2^{122.1}$ quartets remain. The probabilities of Step (A) and (B) are same, thus we have $N^{2} / 2^{130.18}$ candidates for right quartets in Step 3.

The time complexity of the preliminary elimination is as follows: In Step 2, we have $2 N$ memory accesses. In step 4 , the number of analyzed quartets, which is the number of required memory access is $N^{2} / 2^{114.02}$. Note that there is no need to go over all bins. In total $2 N+N^{2} / 2^{114.02}$ memory accesses is required, and thus for $N=2^{106.89}$, the total running time of the preliminary elimination is expected to be $2^{107.89}+2^{99.76}$ memory accesses.

For $N=2^{106.89}$, we have $\left(2^{106.89}\right)^{2} / 2^{130.18}=2^{83.6}$ candidates of right quartets. The time complexity of the attack is dominated by the partial decryptions in Step 2(b) for $\mathrm{j}=0$ in 9 . Therefore, the running time of steps 2 - 4 of the attack is $2^{8} \cdot 2^{83.6} \cdot 1 / 14=2^{87.69}$. The total running time is dominated by Step 1, i.e. $2^{107.89}$ memory accesses. 


\section{Impossible Differential Attack on 16-Round SMS4}

\subsection{Impossible Differential Attack}

Unlike traditional differential cryptanalysis which tracks differences that propagate through the cipher with high probability, impossible differential cryptanalysis exploits differentials with probability zero.

The attack used in 9] is a combination of the general technique called miss in the middle, which is used to construct impossible differential, and the early abort technique which partially determines whether or not a candidate pair is useful. The main idea is to find two characteristics with probability one, whose conditions cannot be met together [4. Then, the key can be found by analyzing the rounds surrounding the impossible event, and guessing the subkeys of these rounds. If the impossible event occurs when a candidate key is used, it is obvious that the suggested key is not the right key.

\subsection{The Previous Attack on 16-Round SMS4}

The attack uses a set of 12-round impossible differentials of the form $\left(e_{\Gamma}, e_{\Gamma}\right.$, $\left.e_{\Gamma}, 0\right) \nrightarrow\left(0, e_{\Gamma}, e_{\Gamma}, e_{\Gamma}\right)$. Two 6 -round differentials with probability one are concatenated for the attack. The first differential used in the construction of the impossible differential is $\left(e_{\Gamma}, e_{\Gamma}, e_{\Gamma}, 0\right) \rightarrow\left(e_{\Gamma}, x_{1}, y_{1}, z_{1}\right)$ and the second differential is $\left(z_{2}, y_{2}, x_{2}, e_{\Gamma}\right) \rightarrow\left(0, e_{\Gamma}, e_{\Gamma}, e_{\Gamma}\right)$, where $x_{i} \in \Theta\left(e_{\Gamma}\right), y_{i} \in \Upsilon\left(e_{\Gamma}, x_{i}\right)$, $z_{i} \in \Pi\left(e_{\Gamma}, x_{i}, y_{i}\right)$ for $i=1,2$. These 12-round differentials are used to conduct an impossible differential attack on SMS4 reduced to 16 rounds by adding two additional rounds before and after the differentials.

The attack uses $\Gamma \subseteq\{0,1, \ldots, 15\}$. Hence, in round 1 , for every $\Gamma$, there are $127^{2}$ input differences that may lead to $e_{\Gamma}$ as the output difference of $\mathrm{T}$, and they can be generated by $127^{6}$ input differences in round 0 , which is denoted by the set $\Sigma_{1}(\Gamma)$ for each $\Gamma$. Similarly, there are $127^{2}$ output differences after round 14 , that can be generated by $e_{\Gamma}$, and they cause $127^{6}$ possible output differences after round 15 which, is denoted by the set $\Sigma_{2}(\Gamma)$ for each $\Gamma 6$

The attack procedure of 9 is as follows:

1. Choose $2^{9}$ structures of $2^{96}$ plaintexts each where the most significant 2 bytes of the two rightmost words of the plaintexts in each structure is fixed. (Thus, each structure generates $\left(2^{96}\right)^{2} / 2=2^{191}$ plaintext pair.7 $\left(P_{i}, P_{j}\right)$ with the desired input difference $\left.\left(*, *, e_{\Gamma}, e_{\Gamma}\right)\right)$.

(a) Obtain the corresponding ciphertext pairs of the structures.

(b) Choose the pairs that satisfy both $P_{i} \oplus P_{j} \in \Sigma_{1}(\Gamma)$ and $C_{i} \oplus C_{j} \in \Sigma_{2}(\Gamma)$ simultaneously for the same $\Gamma$, for all possible $\Gamma$ 's.

2. For all the remaining ciphertext pairs $\left(C_{i}, C_{j}\right)$ :

\footnotetext{
${ }^{6}$ In [9], these sets are denoted by $\Sigma_{1}$ and $\Sigma_{2}$. But actually they are not independent of the choice of $\Gamma$. Since the attack procedure runs over all possible such $\Gamma$ 's, it is more clear and more accurate to denote these sets as a function of $\Gamma$.

${ }^{7}$ In 9 , it was claimed that each structure proposes only $2^{190}$ plaintext pairs.
} 
Table 4. The two 6-round differentials

\begin{tabular}{|c|cccc|}
\hline Round & $\Delta X_{i .0}$ & $\Delta X_{i .1}$ & $\Delta X_{i .2}$ & $\Delta X_{i .3}$ \\
\hline 0 & $e_{\Gamma}$ & $e_{\Gamma}$ & $e_{\Gamma}$ & 0 \\
1 & $e_{\Gamma}$ & $e_{\Gamma}$ & 0 & $e_{\Gamma}$ \\
2 & $e_{\Gamma}$ & 0 & $e_{\Gamma}$ & $e_{\Gamma}$ \\
3 & 0 & $e_{\Gamma}$ & $e_{\Gamma}$ & $e_{\Gamma}$ \\
4 & $e_{\Gamma}$ & $e_{\Gamma}$ & $e_{\Gamma}$ & $x_{1}$ \\
5 & $e_{\Gamma}$ & $e_{\Gamma}$ & $x_{1}$ & $y_{1}$ \\
\hline 6 & $e_{\Gamma}$ & $x_{1}$ & $y_{1}$ & $z_{1}$ \\
6 & $e_{z_{2}}$ & $y_{2}$ & $x_{2}$ & $e_{\Gamma}$ \\
\hline 7 & $y_{2}$ & $x_{2}$ & $e_{\Gamma}$ & $e_{\Gamma}$ \\
8 & $x_{2}$ & $e_{\Gamma}$ & $e_{\Gamma}$ & $e_{\Gamma}$ \\
9 & $e_{\Gamma}$ & $e_{\Gamma}$ & $e_{\Gamma}$ & 0 \\
10 & $e_{\Gamma}$ & $e_{\Gamma}$ & 0 & $e_{\Gamma}$ \\
11 & $e_{\Gamma}$ & 0 & $e_{\Gamma}$ & $e_{\Gamma}$ \\
12 & 0 & $e_{\Gamma}$ & $e_{\Gamma}$ & $e_{\Gamma}$ \\
\hline
\end{tabular}

(a) Compute the 4-byte difference just before the $\mathrm{L}$ transformation in round 15 , and denote it by $\Delta_{i, j}^{15}$ (i.e., $\Delta_{i, j}^{15}=L^{-1}\left(C_{i, 3} \oplus C_{j, 3}\right)$ ).

(b) For $l=0$ to 3 :

i. Guess the $l$-th byte of the subkey $R K_{15}$ and partially decrypt $\left(C_{i}, C_{j}\right)$ to get the $l$-th byte of the difference just after the $\mathrm{S}$ transformation in round 15 , denote them by $\left(T_{i, l}, T_{j, l}\right)$.

ii. Check if $T_{i, l} \oplus T_{j, l}=\Delta_{i, j, l}^{15}$ and keep the pairs that satisfy the equality.

3. For all the remaining pairs $\left(T_{i}, T_{j}\right)$ :

(a) Compute the 4-byte difference just before the $\mathrm{L}$ transformation in round 14 and denote it by $\Delta_{i, j}^{14}$.

(b) For $l=0$ to 1 :

i. Guess the $l$-th byte of the subkey $R K_{14}$ and partially decrypt $\left(T_{i}, T_{j}\right)$ to get the $l$-th byte of their intermediate values just after the $\mathrm{S}$ transformation in round 14 , denote them by $\left(Q_{i, l}, Q_{j, l}\right)$.

ii. Check if $Q_{i, l} \oplus Q_{j, l}=\Delta_{i, j, l}^{14}$ and keep the pairs that satisfy the equality.

4. For all plaintext pairs $\left(P_{i}, P_{j}\right)$ corresponding to the remaining ciphertexts after Step 3:

(a) Compute the 4-byte difference just before the $\mathrm{L}$ transformation in round 0 and denote by it $\Delta_{i, j}^{0}$.

(b) For $l=0$ to 3 :

i. Guess the $l$-th byte of the subkey $R K_{0}$ and partially encrypt $\left(P_{i}, P_{j}\right)$ to get the $l$-th byte of their intermediate values just after the $\mathrm{S}$ transformation in round 0 , denote them by $\left(R_{i, l}, R_{j, l}\right)$.

ii. Check if $R_{i, l} \oplus R_{j, l}=\Delta_{i, j, l}^{0}$ and keep the pairs that satisfy the equality.

5. For all the remaining pairs $\left(R_{i}, R_{j}\right)$ : 
(a) Compute the 4-byte difference just before the $\mathrm{L}$ transformation in round 1 and denote by $\Delta_{i, j}^{1}$.

(b) For $l=0$ to 1 :

i. Guess the $l$-th byte of the subkey $R K_{1}$ and partially encrypt $\left(R_{i}, R_{j}\right)$ to get the $l$-th byte of their intermediate values just after the $\mathrm{S}$ transformation in round 1 . Denote them by $\left(S_{i, l}, S_{j, l}\right)$.

ii. Check if $S_{i, l} \oplus S_{j, l}=\Delta_{i, j, l}^{1}$. If there exists a qualified pair then discard the guess of 96 subkey bits and try another, otherwise proceed to the next step.

6. Guess the user key from the known subkey values, and perform a trial encryption. If a key is suggested then output it. Otherwise, continue with a new guess of $R K_{15}$ (i.e., go to Step 2).

The claimed time complexity of this attack is of $2^{107} 16$-round SMS4 computations in [9] and it requires $2^{105}$ chosen plaintexts.

\subsection{Fixing and Improving the 16-Round Attack}

Like in the rectangle attack of [9], in the impossible differential attack of [9], the time complexity analysis is also calculated only for candidates of right pairs after preliminary elimination (the pairs which enter Step 2), and it does not include the time complexity of the first elimination itself. Also, the data complexity suggested in [9] is too low.

Data Complexity Issues: Each structure is composed of $2^{96}$ plaintexts of the form $\left(*, *,\left(a, b_{i}\right),\left(c, d_{i}\right)\right)$ where $*$ denotes all possible values and $a, c$ denote the chosen constants of the structure, i.e., each structure suggests $\left(2^{96}\right)^{2} / 2=2^{191}$ pairs. In order to have the desired input difference $\left(*, *, e_{\Gamma}, e_{\Gamma}\right)$, we must have $b_{i} \oplus b_{j}=d_{i} \oplus d_{j}=\tilde{e_{\Gamma}}$ where $\tilde{e_{\Gamma}}$ is the least significant two bytes of $e_{\Gamma}$ for each $\Gamma$.

In a structure, for each $\left(b_{i}, b_{j}, d_{i}, d_{j}\right)$, there are $2^{64} \cdot 2^{64}=2^{128}$ possible pairs of plaintexts. There are $2^{16} \cdot 2^{16} / 2=2^{31}$ possible $\left(b_{i}, b_{j}\right)$ pairs, and for each pair there exists $2^{16}$ possible $d_{i}$ 's. Given $\left(b_{i}, b_{j}\right)$ and $d_{i}$, there exists a unique $d_{j}$ value satisfying the above condition. Hence, only $2^{128} \cdot 2^{31} \cdot 2^{16}=2^{175}$ of the $2^{191}$ pairs satisfy the desired input difference.

$\Sigma_{1}(\Gamma)$ is composed of $127^{6} \simeq 2^{42}$ possible input differences for each $\Gamma$. Therefore, the probability of a pair to have $P_{1} \oplus P_{2} \in \Sigma_{1}(\Gamma)$ is $2^{42} / 2^{64}=2^{-22}$, and $2^{175} \cdot 2^{-22}=2^{153}$ pairs pass this step. Note that once the plaintext pair is fixed, $\Gamma$ is also fixed, so does $\Sigma_{1}(\Gamma)$ and $\Sigma_{2}(\Gamma)$. Similar to $\Sigma_{1}(\Gamma), \Sigma_{2}(\Gamma)$ is composed of $127^{6} \simeq 2^{42}$ possible output differences for each $\Gamma$. Therefore, the probability of a pair to have $C_{1} \oplus C_{2} \in \Sigma_{2}(\Gamma)$ is $2^{42} / 2^{128}=2^{-84}$ and the number of pairs for a given structure passing the Step 1 of the algorithm is $2^{153} \cdot 2^{-84}=2^{69}$.

Starting with $S$ such structures, the number of plaintext pairs passing the preliminary elimination is $S \cdot 2^{69}$. The probability that a given subkey is discarded by a given structure is thus, $2^{69} \cdot\left(2^{-7}\right)^{12}=2^{-15}$, and that it is not discarded by all $S$ structures is $\left(1-2^{-15}\right)^{S}$. In order to discard all wrong subkeys, we need 
to make sure that the probability of a wrong key to remain is about $2^{-96}$, i.e., $2^{-96}=1-\left(1-2^{-15}\right)^{S}$. Thus for $S=2^{9}$, it is not probable to discard most of the subkey guesses.

The number of required structures can be calculated as follows: There are $2^{96}$ possible subkeys, and $S \cdot 2^{-15}$ pairs are expected for each subkey. In order to have all wrong subkeys with one pair (i.e., suggested by some pair and thus identified as wrong ones), the probability of a wrong key to have no pairs should be less than $2^{-96}$. The probability of having no pairs is $e^{-S \cdot 2^{-15}}$. Solving this, we obtain that $S=2^{21.06}$ structures are needed for the attack.

Algorithm for the detection of candidate pairs: Denote a plaintext by $P_{i}=\left(P_{i, 0}, P_{i, 1},\left(a_{i}, b_{i}\right),\left(c_{i}, d_{i}\right)\right)$, a ciphertext by $C_{i}=\left(\left(w_{i}, x_{i}\right),\left(y_{i}, z_{i}\right), C_{i, 2}, C_{i, 3}\right)$.

1. Insert every plaintext-ciphertext pair $\left(P_{i}, C_{i}\right)$ of each structure, indexed by the least significant 2 bytes of the rightmost two words of the plaintext and the most significant two words of the corresponding ciphertext (i.e, $\left.b_{i}\left\|d_{i}\right\| w_{i}\left\|x_{i}\right\| y_{i} \| z_{i}\right)$ into a hash table.

2. For each $\tilde{e}_{\Gamma}$ :

(a) For every non-empty bin satisfying $b_{i}<b_{j}$ :

i. go to the corresponding bin:

$$
\begin{aligned}
& b_{i}\left\|d_{i}\right\| w_{i}\left\|x_{i}\right\| y_{i}\left\|z_{i} \oplus \tilde{e_{\Gamma}}\right\| \tilde{e_{\Gamma}}\left\|e_{\Gamma}\right\| e_{\Gamma}=b_{j}\left\|d_{j}\right\| w_{j}\left\|x_{j}\right\| y_{j} \| z_{j} \\
& \text { (i.e. } w_{i}=w_{j} \text { and } y_{i}=y_{j} \text { ). }
\end{aligned}
$$

ii. For all possible combinations of entries, pick the plaintext pairs for which:
A. $P_{i, 1} \oplus P_{j, 1} \in \theta\left(e_{\Gamma}\right)$
B. $C_{i, 2} \oplus C_{j, 2} \in \theta\left(e_{\Gamma}\right)$
C. $P_{i, 0} \oplus P_{j, 0} \in \Upsilon\left(e_{\Gamma}, P_{i, 1} \oplus P_{j, 1}\right)$
D. $C_{i, 3} \oplus C_{j, 3} \in \Upsilon\left(e_{\Gamma}, C_{i, 2} \oplus C_{j, 2}\right)$

is satisfied. (If one of them fails, do not check the remaining conditions.)

3. If any pair satisfying (A)-(D) is found, analyze it in Steps 2-6 of the attack.

The time complexity of the preliminary elimination is as follows: In Step 1, we have $2^{96}$ memory accesses for each structure. There are $2^{96}$ plaintext-ciphertext pairs in a structure, therefore, the expected number of entries in each of the $2^{96}$ bins is 1 . The resulting number of required memory accesses for Step 2 is $2^{16} \cdot 2^{96} / 2=2^{111}$ for a given structure. Therefore, the total number of memory accesses of the algorithm is $S \cdot 2^{111}=2^{132.06}$.

As mentioned earlier in data complexity issues, the number of pairs passing the preliminary elimination is $2^{69}$ per structure. Therefore, starting with $S=2^{21.06}$ structures, which results in $2^{117.06}$ chosen plaintexts, the number of plaintext pairs passing the preliminary elimination is $2^{21.06} \cdot 2^{69}=2^{90.06}$. The time complexity of the partial encryptions/decryptions in Steps 2(b), 3(b), 4(b) and $5(\mathrm{~b})$ of the algorithm is:

$$
\sum_{i=1}^{12}\left(2^{91.06} \cdot \frac{1}{127^{i-1}} \cdot 2^{8}\right) \cdot \frac{1}{16}=2^{95.07}
$$

However, the time complexity of the attack is dominated by the $2^{117.06}$ partial encryptions required to obtain the ciphertext pairs in Step 1, and by the $2^{132.06}$ memory accesses performed for the preliminary elimination. 


\section{Summary}

In this paper, we reviewed the rectangle attack on 14-rounds and impossible differential attack on 16-rounds SMS4 presented by Lu. We identified some flaws in the attack algorithms and in the time and data complexity analysis of these attacks. We then followed by correcting and improving these attacks.

We first showed that a better 12-round rectangle distinguisher with probability $2^{-209.78}$ can be found, reducing the amount of required chosen plaintexts to perform the attack from $2^{121.82}$ to $2^{107.89}$. Then, we presented a more efficient algorithm to perform the preliminary elimination.

We also identified some flaws in the previous impossible differential attack of 9 . We first showed that more data is needed for the analysis, and we also presented a more efficient algorithm for the preliminary elimination. The results are summarized in Table 5 .

Table 5. Comparison of the Results for the Existing Attacks

\begin{tabular}{|l|c|cc|c|}
\hline \multicolumn{1}{|c|}{ Attack Type } & \#of Rounds & \multicolumn{2}{|c|}{ Complexity } & Source \\
\hline Integral Attack & & Data & Time $^{a}$ & \\
Rectangle Attack & 13 & $2^{16}$ & $2^{114}$ Enc & {$[7]$} \\
Impossible Differential Attack & 14 & $2^{121.82, b}$ & $2^{116.66, b}$ Enc & {$[\underline{9]}$} \\
\hline Rectangle Attack [New] & 16 & $2^{105, b}$ & $2^{107, b}$ Enc & {$[9]$} \\
Impossible Differential Attack [New] & 14 & $2^{107.89}$ & $2^{107.89} \mathrm{MA}$ & Section [3.2 \\
\hline Rectangle Attack & 16 & $2^{117.06}$ & $2^{132.06} \mathrm{MA}$ & Section [4.3 \\
Boomerang Attack & 18 & $2^{125}$ & $2^{116}$ Enc & {$[12]$} \\
Rectangle Attack & 18 & $2^{120}$ & $2^{116.83}$ Enc & {$[\underline{6}$} \\
Differential Attack & 21 & $2^{118}$ & $2^{112.83}$ Enc & {$\left[\begin{array}{l}126.6 \\
\text { Linear Attack }\end{array}\right.$} \\
Differential Attack & 22 & $2^{117}$ & $2^{109.86}$ Enc & {$[12]$} \\
\hline
\end{tabular}

Enc - Encryptions, MA - Memory Accesses.

${ }^{a}$ Time complexities are calculated only for the given algorithms, and they do not include the complexity of obtaining the required data for the attack, which may be higher.

${ }^{b}$ As noted in Sections 3.1 and 4.3 these figures are underestimated.

\section{References}

1. Beijing Data Security Technology Co. Ltd, Specification of SMS4 (2006) (in Chinese), http://www.oscca.gov.cn/UpFile/200621016423197990.pdf

2. Biham, E., Dunkelman, O., Keller, N.: The Rectangle Attack Rectangling the Serpent. In: Pfitzmann, B. (ed.) EUROCRYPT 2001. LNCS, vol. 2045, pp. 340-357. Springer, Heidelberg (2001)

3. Biham, E., Dunkelman, O., Keller, N.: New Results on Boomerang and Rectangle Attacks. In: Daemen, J., Rijmen, V. (eds.) FSE 2002. LNCS, vol. 2365, pp. 1-16. Springer, Heidelberg (2002) 
4. Biham, E., Birjukov, A., Shamir, A.: Miss in the Middle Attacks on IDEA and Khufu. In: Knudsen, L.R. (ed.) FSE 1999. LNCS, vol. 1636, pp. 124-138. Springer, Heidelberg (1999)

5. Kelsey, J., Kohno, T., Schneier, B.: Amplified Boomerang Attacks Against Reduced-Round MARS and Serpent. In: Schneier, B. (ed.) FSE 2000. LNCS, vol. 1978, pp. 75-93. Springer, Heidelberg (2001)

6. Kim, T., Kim, J., Hong, S., Sung, J.: Linear and Differential Cryptanalysis of Reduced SMS4 Block Cipher, Cryptology ePrint Archive: Report 2008/281 (2008)

7. Knudsen, L.R., Wagner, D.: Integral Cryptanalysis. In: Daemen, J., Rijmen, V. (eds.) FSE 2002. LNCS, vol. 2365, pp. 112-127. Springer, Heidelberg (2002)

8. Liu, F., et al.: Analysis of the SMS4 Block Cipher. In: Pieprzyk, J., Ghodosi, H., Dawson, E. (eds.) ACISP 2007. LNCS, vol. 4586, pp. 158-170. Springer, Heidelberg (2007)

9. Lu, J.: Attacking Reduced-Round Versions of the SMS4 Block Cipher in the Chinese WAPI Standart. In: Qing, S., Imai, H., Wang, G. (eds.) ICICS 2007. LNCS, vol. 4861, pp. 306-318. Springer, Heidelberg (2007)

10. Wagner, D.: The Boomerang Attack. In: Knudsen, L.R. (ed.) FSE 1999. LNCS, vol. 1636, pp. 156-170. Springer, Heidelberg (1999)

11. Zhang, L., Wu, W.: Differential Fault Attack on SMS4. Chinese Journal of Computers 29(9) (2006)

12. Zhang, L., Zhang, W., Wu, W.: Cryptanalysis of Reduced-Round SMS4 Block Cipher. In: Mu, Y., Susilo, W., Seberry, J. (eds.) ACISP 2008. LNCS, vol. 5107, pp. 216-229. Springer, Heidelberg (2008) 